\title{
Pull-back of singular Levi-flat hypersurfaces
}

\author{
Andrés Beltrán, Arturo Fernández-Pérez and Hernán Neciosup
}

\begin{abstract}
We study singular real analytic Levi-flat subsets invariant by singular holomorphic foliations in complex projective spaces. We give sufficient conditions for a real analytic Levi-flat subset to be the pull-back of a semianalytic Levi-flat hypersurface in a complex projective surface under a rational map or to be the pull-back of a real algebraic curve under a meromorphic function. In particular, we give an application to the case of a singular real analytic Levi-flat hypersurface. Our results improve previous ones due to Lebl and Bretas-Fernández-Pérez-Mol.
\end{abstract}

\section{Introduction and statement of the results}

Let $M$ be a complex manifold of $\operatorname{dim}_{\mathbb{C}} M=N \geq 2$, a closed subset $H \subset M$ is a real analytic subvariety if for every $p \in H$, there are real analytic functions with real values $\varphi_{1}, \ldots, \varphi_{k}$ defined in a neighborhood $U \subset M$ of $p$, such that $H \cap U$ is equal to the set where all $\varphi_{1}, \ldots, \varphi_{k}$ vanish. A complex subvariety is precisely the same notion, considering holomorphic functions instead of real analytic functions. We say that a real analytic subvariety $H$ is irreducible if whenever we write $H=H_{1} \cup H_{2}$ for two subvarieties $H_{1}$ and $H_{2}$ of $M$, then either $H_{1}=H$ or $H_{2}=H$. If $H$ is irreducible, it has a well-defined dimension $\operatorname{dim}_{\mathbb{R}} H$. Let $H_{\text {reg }}$ denote its regular part, i.e., the subset of points near which $H$ is a real analytic submanifold of dimension equal to $\operatorname{dim}_{\mathbb{R}} H$. A set is semianalytic if it is locally constructed from real analytic sets by finite union, finite intersection, and complement. For a real analytic subvariety $H$, the set $\overline{H_{\text {reg }}}$ is a semianalytic subset where the closure is with the standard topology. In general, the inclusion $\overline{H_{\text {reg }}} \subset H$ is proper, which happens, for instance in the

This work was supported by the Pontifícia Universidad Católica del Perú project VRIDGI-2018-0024. The second author is partially supported by CNPq-Brazil Grant Number $302790 / 2019-5$.

Key words and phrases: Levi-flat subsets, holomorphic foliations. 2010 Mathematics Subject Classification: primary 32V40, $32 \mathrm{~S} 65$. 
Whitney umbrella. We really only study the set $\overline{H_{\text {reg }}}$, in this sense, we consider $\operatorname{Sing}(H):=\overline{H_{\text {reg }}} \backslash H_{\text {reg }}$ as the singular set of $H$, this is not the usual definition of the singular set in the literature, see for instance [15].

If $H \subset M$ is a real analytic hypersurface i.e., a real analytic subvariety of real codimension one, then for each $p \in H_{\text {reg }}$, there is a unique complex hyperplane $\mathscr{L}_{p} \subset T_{p} H_{\text {reg. }}$. This defines a real analytic distribution $p \mapsto \mathscr{L}_{p}$ of complex hyperplanes in $T H_{\text {reg. }}$. When this distribution is integrable in the sense of Frobenius, we say that $H$ is Levi-flat. Here, $H_{\text {reg }}$ is foliated by codimension one immersed complex submanifolds. This foliation, denoted by $\mathscr{L}$, is known as Levi foliation. According to Cartan [4], $\mathscr{L}$ can be extended to a non-singular holomorphic foliation in a neighborhood of $H_{\text {reg }}$ in $M$, but in general, it is not possible to extend $\mathscr{L}$ to a singular holomorphic foliation in a neighborhood of $H$. There are examples of singular Levi-flat hypersurfaces whose Levi foliations extend to singular holomorphic webs in the ambient space, see for instance [8] and [21]. When there is a singular holomorphic foliation $\mathscr{F}$ in the ambient space $M$ that coincide with the Levi foliation on $H_{\text {reg }}$, we say either that $H$ is invariant by $\mathscr{F}$ or that $\mathscr{F}$ is tangent to $H$. Cerveau and Lins Neto [6] proved that germs of singular foliations of codimension one at $\left(\mathbb{C}^{N}, 0\right)$ tangent to real analytic Levi-flat hypersurfaces have meromorphic (possibly holomorphic) first integrals. We recall that a non-constant function $f$ is the first integral for a foliation $\mathscr{F}$ if each leaf of $\mathscr{F}$ is contained in a level set of $f$. In the global context, the same problem has been studied in [1] and [9].

The aim of this paper is to study holomorphic foliations tangent to real analytic Levi-flat subsets in complex manifolds. An irreducible real analytic subvariety $H \subset$ $M$, where $M$ is an $N$-dimensional complex manifold, $N \geq 2$, is a Levi-flat subset if it has real dimension $2 n+1$ and its regular part $H_{r e g}$ is foliated by immersed complex manifolds of complex dimension $n$. Similarly to the case of hypersurfaces, this foliation is called Levi foliation of $H$ and will be denoted by $\mathscr{L}$. The number $n$ is the Levi dimension of $H$. We use the qualifier "Levi" for the foliation, its leaves, and its dimension. Since we deal with real analytic Levi-flat subsets in complex manifolds we shall consider that $H$ is coherent. Coherence implies that $H$ admits a global complexification [11, p. 40]. Here coherent means that its ideal sheaf $\mathcal{I}(H)$ in $\mathcal{A}_{\mathbb{R}, M}$, the sheaf of germs of real analytic functions with real values in $M$, is a coherent sheaf of $\mathcal{A}_{\mathbb{R}, M}$-modules. It follows from Oka's theorem [17, p. 94, Proposition 5] that $H$ is coherent if the sheaf $\mathcal{I}(H)$ is locally finitely generated, the latter means that for every point $p \in H$ there exists an open neighborhood $U \subset M$ and a finite number of functions $\varphi_{j}$, real analytic in $U$ and vanishing on $H$, such that for any $q \in U$, the germs of $\varphi_{j}$ at $q$ generate the ideal $\mathcal{I}\left(H_{q}\right)$, where $H_{q}$ is the germ of $H$ at $q$. We remark that not every real analytic subset is coherent as we shall see in Section 3 of this paper. 
In [3], singular Levi-flat subsets appear in the result of the lifting of a real analytic Levi-flat hypersurface to the projectivized cotangent bundle of the ambient space through the Levi foliation and in [20], the authors gave a complete characterization of dicritical singularities of local Levi-flat subsets in terms of their Segre varieties.

Let $Y$ be a complex projective surface, $T \subset Y$ be a real analytic Levi-flat hypersurface, $X \subset \mathbb{P}^{N}, N \geq 3$, be a complex projective subvariety of complex dimension $k<N$ and $\rho: X \rightarrow Y$ be a dominant rational map. Then it is easy to show that $H=\overline{\rho^{-1}(T)}$ is a real analytic Levi-flat subset in $\mathbb{P}^{N}$ and so $H$ is a Levi-flat subset defined via pull-back. Therefore, one natural question is:

Given a real analytic Levi-flat subset $H \subset \mathbb{P}^{N}$. Under what condition, $H$ is given by the pull-back of a Levi-flat hypersurface in a projective complex surface via a rational map?

In [14], Lebl gave sufficient conditions for a real analytic Levi-flat hypersurface in $\mathbb{P}^{N}$ to be a pull-back of a real algebraic curve in $\mathbb{C}$ via a meromorphic function. In [2], Bretas et al. proved an analogous result for real analytic Levi-flat subsets in $\mathbb{P}^{N}$. The main hypothesis in these articles is that the Levi foliation has infinitely many algebraic leaves. In this paper, we give an answer to the question, assuming that $H$ is invariant by a singular holomorphic foliation on $\mathbb{P}^{N}$ with quasi-invariant subvarieties (see Section 2). An irreducible complex subvariety $S \subset X$ of complex dimension $n$ is quasi-invariant by a global $n$-dimensional foliation $\mathscr{F}$ on a complex projective manifold $X$ if it is not $\mathscr{F}$-invariant, but the restriction to the foliation $\mathscr{F}$ to $S$ is an algebraically integrable foliation of dimension $n-1$, i.e. every leaf of $\left.\mathscr{F}\right|_{S}$ is algebraic. The concept of quasi-invariant subvarieties was introduced by Pereira-Spicer [19] for codimension one holomorphic foliations on complex projective manifolds to prove a variant of the classical Darboux-Jouanolou Theorem. Here we shall use this concept for Levi foliations to prove our main result:

Theorem 1.1. Let $H \subset \mathbb{P}^{N}, N \geq 3$, be an irreducible real analytic Levi-flat subset of Levi dimension $n$ invariant by an $n$-dimensional singular holomorphic foliation $\mathscr{F}$ on $\mathbb{P}^{N}$. Suppose that $H$ is coherent and $n>N / 2$. If the Levi foliation has infinitely many quasi-invariant subvarieties of complex dimension $n$, then there exists a unique projective subvariety $X$ of complex dimension $n+1$ containing $H$ such that either there exists a rational map $R: X \rightarrow \rightarrow \mathbb{P}^{1}$, and real algebraic curve $C \subset \mathbb{P}^{1}$ such that $\overline{H_{\text {reg }}} \subset \overline{R^{-1}(C)}$ or there exists a dominant rational map $\rho: X-\rightarrow Y$ on a projective surface $Y$ and a semianalytic Levi-flat subset $T \subset Y$ such that $\overline{H_{\text {reg }}} \subset \overline{\rho^{-1}(T)}$.

We emphasize that the hypothesis $n>N / 2$ implies that $H$ is necessarily a real analytic subvariety with singularities. In fact, Ni-Wolfson [18, Theorem 2.4] proved 
that no nonsingular real analytic Levi-flat subset of the Levi dimension $n$ exist in $\mathbb{P}^{N}, n>N / 2$.

Applying Theorem 1.1 to $n=N-1$, we get the following corollary:

Corollary 1.2. Let $H \subset \mathbb{P}^{N}, N \geq 3$, be an irreducible coherent real analytic Levi-flat hypersurface invariant by a codimension one holomorphic foliation $\mathscr{F}$ on $\mathbb{P}^{N}$. If the Levi foliation has infinitely many quasi-invariant complex hypersurfaces, then either there exists a rational map $R: \mathbb{P}^{N}-\rightarrow \mathbb{P}^{1}$, and real algebraic curve $C \subset \mathbb{P}^{1}$ such that $\overline{H_{\text {reg }}} \subset \overline{R^{-1}(C)}$ or there exists a dominant rational map $\rho: \mathbb{P}^{N} \rightarrow Y$ on a projective surface $Y$ and a semianalytic Levi-flat subset $T \subset Y$ such that $\overline{H_{r e g}} \subset$ $\overline{\rho^{-1}(T)}$.

When $H$ is a real analytic hypersurface, the above corollary gives a nice characterization of coherent real analytic Levi-flat hypersurfaces in $\mathbb{P}^{N}, N \geq 3$, invariant by codimension one holomorphic foliations which admit infinitely many quasi-invariant complex hypersurfaces. Observe that, in order to improve our results, we need to extend the Levi foliation of a Levi-flat subset to a holomorphic foliation in the ambient space. Therefore, another interesting question is:

Given a real analytic Levi-flat subset $H \subset \mathbb{P}^{N}$ with Levi foliation $\mathcal{L}$. Under what condition, $\mathcal{L}$ extend to a singular holomorphic foliation on $\mathbb{P}^{N}$ ?

When $H$ is a local real analytic Levi-flat hypersurface, Lebl solved the above question in the non-dicritical case in [15].

The paper is organized as follows: in Section 2, we define the concept of quasiinvariant subvarieties of a foliation with complex leaves and state the main result of [19], such a result is key to prove Theorem 1.1. Section 3 is devoted to the study of real analytic Levi-flat subset in complex manifolds, using some results of [3] and [2], we prove the algebraic extension of the intrinsic complexification of $H$. In Section 4 , we prove Theorem 1.1 and in Section 5 we prove Corollary 1.2. Finally, in Section 6, we give two examples. The first is an example of a Levi-flat hypersurface where Theorem 1.1 applies. In the second example, we construct a Levi-flat hypersurface in $\mathbb{P}^{3}$ that is not a pull-back of a Levi-flat hypersurface of $\mathbb{P}^{2}$ under a rational map. Moreover, this example also is not a pull-back of a real algebraic curve under a meromorphic function.

\section{Foliations with complex leaves and quasi-invariant subvarieties}

\subsection{Foliations with complex leaves}

A foliation with complex leaves of complex dimension $n$ is a smooth foliation $\mathcal{G}$ of dimension $2 n$ whose local models are domains $U=W \times B$ of $\mathbb{C}^{n} \times \mathbb{R}^{k}, W \subset \mathbb{C}^{n}$, 
$B \subset \mathbb{R}^{k}$ and whose local transformations are of the form

$$
\varphi(z, t)=(f(z, t), h(t))
$$

where $f$ is holomorphic with respect to $z$. A domain $U$ as above is said to be a distinguished coordinate domain of $\mathcal{G}$ and $z=\left(z_{1}, \ldots, z_{n}\right), t=\left(t_{1}, \ldots, t_{k}\right)$ are said to be distinguished local coordinates. As examples of such foliations we have the Levi foliations of Levi-flat hypersurfaces of $\mathbb{C}^{n}$, see for instance [5] and [10].

If we replace $\mathbb{R}^{k}$ by $\mathbb{C}^{k}$ and in (1) we assume $t \in \mathbb{C}^{k}$ and that $f, h$ are holomorphic with respect to $z, t$ then we get the notion of holomorphic foliation of complex codimension $k$.

Now we define foliations with singularities. Let $M$ be a complex manifold. A singular foliation with complex leaves $\mathcal{G}$ of dimension $n$ on $M$ is a foliation with complex leaves of dimension $n$ on $M \backslash E$, where $E$ is a real analytic subvariety of $M$ of real dimension $<2 n$. A point $p \in E$ is called a removable singularity of $\mathcal{G}$ of there is a chart $(U, \varphi)$ around $p$, compatible with the atlas $\mathcal{A}$ of $\mathcal{G}$ restricted to $M \backslash E$, in the sense that $\varphi \circ \varphi_{i}^{-1}$ and $\varphi_{i} \circ \varphi^{-1}$ have the form (1) for all $\left(U_{i}, \varphi_{i}\right) \in \mathcal{A}$ with $U \cap U_{i} \neq \varnothing$. The set of non-removable singularities of $\mathcal{G}$ in $E$ is called the singular set of $\mathcal{G}$, and is denoted by $\operatorname{Sing}(\mathcal{G})$.

\subsection{Quasi-invariant subvarieties}

Let $Z$ be a projective manifold of complex dimension $N \geq 2$ and let $\mathscr{G}$ be a foliation with complex leaves of dimension $n$ on $Z$.

Definition 2.1. We say that $\mathscr{G}$ is an algebraically integrable foliation on $Z$ if every leaf of $\mathscr{G}$ is algebraic, i.e. every leaf of $\mathscr{G}$ is a projective complex subvariety in $Z$.

Motivated by [19], we define the concept of a subvariety quasi-invariant by a real analytic foliation with complex leaves.

Definition 2.2. An irreducible subvariety $S \subset Z$ of complex dimension $n$ is quasiinvariant by a foliation $\mathscr{G}$ if it is not $\mathscr{G}$-invariant, but the restriction of the foliation $\mathscr{G}$ to $S$ is an algebraically integrable foliation.

We note that the restriction foliation $\left.\mathscr{G}\right|_{S}$ is a codimension one foliation on $S$ and when $\left.\mathscr{G}\right|_{S}$ is an algebraically integrable foliation, we have that every leaf of $\left.\mathscr{G}\right|_{S}$ are projective complex hypersurfaces in $S$. Codimension one holomorphic foliations on $Z$ which admit infinitely many quasi-invariant hypersurfaces have been studied in [19] and its main result is the following. 
Theorem 2.3. (Pereira-Spicer [19]) Let $\mathscr{F}$ be a codimension one holomorphic foliation on a projective manifold $Z$. If $\mathscr{F}$ admits infinitely many quasi-invariant hypersurfaces then either $\mathscr{F}$ is an algebraically integrable foliation, or $\mathscr{F}$ is a pull-back of a foliation of dimension one on a projective surface under a dominant rational map.

\section{Real analytic subsets}

\subsection{Coherent real analytic subsets.}

We present some of the fundamental results concerning coherent real analytic subsets.

Let $H$ be a real analytic subset in an open set $U \subset \mathbb{C}^{n}$ and let $\mathcal{I}(H)$ be its ideal sheaf, it is the sheaf of germs of real analytic functions with real values vanishing on $H$.

Definition 3.1. $H$ is said to be coherent if $\mathcal{I}(H)$ is a coherent sheaf of $\mathcal{A}_{\mathbb{R}, U}$-modules, where $\mathcal{A}_{\mathbb{R}, U}$ is the sheaf of germs of real analytic functions with real values in $U$.

Proposition 3.2. ([17, p. 95]) If $H$ is a coherent real analytic subset and the germ $H_{p}$ of $H$ at $p$ is irreducible, then for $q$ near $p$, we have

$$
\operatorname{dim}_{\mathbb{R}} H_{p}=\operatorname{dim}_{\mathbb{R}} H_{q} .
$$

It is well known that locally, a real analytic subset always admits a complexification (see for instance [11, p. 40]) and it is not true for global real analytic subsets. It is shown in $[11$, p. 54$]$ that the global complexification of a coherent real analytic subset in a complex manifold always exists.

Theorem 3.3. ([11, p. 54]) A real analytic subset in a complex manifold is coherent if and only if it admits a global complexification.

Now we build an irreducible real analytic hypersurface in $\mathbb{P}^{3}$ which is not coherent. Let $\left[z_{0}: z_{1}: z_{2}: z_{3}\right]$ be the homogeneous coordinates in $\mathbb{P}^{3}$ and set $H \subset \mathbb{P}^{3}$ be the complex cone whose equation is

$$
H=\left\{\left(z_{3} \bar{z}_{0}+\bar{z}_{3} z_{0}\right)\left(\left(z_{1} \bar{z}_{0}+\bar{z}_{1} z_{0}\right)^{2}+\left(z_{2} \bar{z}_{0}+\bar{z}_{2} z_{0}\right)^{2}\right)-\left(z_{1} \bar{z}_{0}+\bar{z}_{1} z_{0}\right)^{3}=0\right\} .
$$

The germ $H_{p}$ of $H$ at $p=[1: 0: 0: 0]$ is irreducible and of real dimension 5 at $p$. However, in a neighborhood of $[1: 0: 0: z], z \neq 0, H$ reduces to the complex line $z_{1}=$ $z_{2}=0$, which is of real dimension 2. By Proposition 3.2, it follows that $H$ is not coherent. 


\subsection{Levi-flat subset in complex manifolds.}

We give a brief resume of definitions and some known results about real analytic Levi-flat subsets in complex manifolds. Let $H$ be an irreducible real analytic Leviflat subset of Levi dimension $n$ in an $N$-dimensional complex manifold $M$. The notion of Levi-flat subset germifies and, in general, we do not distinguish a germ at $\left(\mathbb{C}^{N}, 0\right)$ from its realization in some neighborhood $U$ of $0 \in \mathbb{C}^{N}$. If $p \in H_{\text {reg }}$ then, according to [2, Proposition 3.1], there exists a holomorphic coordinate system $z=\left(z^{\prime}, z^{\prime \prime}\right) \in \mathbb{C}^{n+1} \times \mathbb{C}^{N-n-1}$ such that $z(p)=0 \in \mathbb{C}^{N}$ and the germ of $H$ at $p$ is defined by

$$
H=\left\{z=\left(z^{\prime}, z^{\prime \prime}\right) \in \mathbb{C}^{n+1} \times \mathbb{C}^{N-n-1}: \operatorname{Im}\left(z_{n+1}\right)=0, \quad z^{\prime \prime}=0\right\}
$$

where $z^{\prime}=\left(z_{1}, \ldots, z_{n+1}\right)$ and $z^{\prime \prime}=\left(z_{n+2}, \ldots, z_{N}\right)$ and the Levi foliation is given by

$$
\left\{z=\left(z^{\prime}, z^{\prime \prime}\right) \in \mathbb{C}^{n+1} \times \mathbb{C}^{N-n-1}: z_{n+1}=c, \quad z^{\prime \prime}=0 \text {, with } c \in \mathbb{R}\right\} .
$$

This trivial model is, in fact, a local form for a non-singular real analytic Levi-flat subset. Note that in the local form $(2),\left\{z^{\prime \prime}=0\right\}$ corresponds to the unique local $(n+1)$-dimensional complex subvariety of the ambient space containing the germ of $H_{\text {reg }}$ at $p$. These local subvarieties glue together forming a complex variety defined in a whole neighborhood of $H_{r e g}$. It is analytically extendable to a neighborhood of $\overline{H_{\text {reg }}}$ by the following theorem:

Theorem 3.4. (Brunella [3]) Let $M$ be an $N$-dimensional complex manifold and $H \subset M$ be a real analytic Levi-flat subset of Levi dimension $n$. Then, there exists a neighborhood $V \subset M$ of $\overline{H_{\text {reg }}}$ and a unique complex variety $X \subset V$ of dimension $n+1$ containing $H$.

The variety $X$ is the realization in the neighborhood $V$ of a germ of complex analytic variety around $H$. We denote it - or its germ - by $H^{\imath}$ and call it intrinsic complexification or $\imath$-complexification of $H$. It plays a central role in the theory of real analytic Levi-flat subsets. The notion of intrinsic complexification also appears in [22] with the name of the Segre envelope. If $H$ is invariant by a holomorphic foliation on $M$, the same holds for its $z$-complexification, see for instance [2, Proposition 3.3].

Proposition 3.5. Let $H \subset M$ be a real analytic Levi-flat subset of Levi dimension $n$, where $M$ is a complex manifold of dimension $N$. If $H$ is invariant by an $n$-dimensional holomorphic foliation $\mathcal{F}$ on $M$, then its -complexification $H^{\imath}$ is also invariant by $\mathcal{F}$. 
As a consequence, if we denote by $\mathscr{F}^{\imath}:=\left.\mathscr{F}\right|_{H^{\imath}}$ (the restriction of $\mathscr{F}$ to $H^{\imath}$ ), we have $\mathscr{F}^{\imath}$ has codimension one in $H^{\imath}$. The following proposition shows the importance of the assumption of the coherence of a Levi-flat subset.

Proposition 3.6. ([2, Proposition 3.6]) Let $M$ be an $N$-dimensional complex manifold and $H \subset M$ be an irreducible real analytic Levi-flat subset of Levi dimension $n$. Suppose that $H$ is coherent. Then, there exist an open neighborhood $V \subset M$ of $H$ and a unique irreducible complex subvariety $X$ of $V$ of complex dimension $n+1$ containing $H$.

The variety $X$ is the small variety of complex dimension $n+1$ that contains $H$. Again, let us denote this variety by $H^{\imath}$, the intrinsic complexification of $H$.

\subsection{Levi-flat subsets in complex projective spaces}

In this subsection, we state some results of real analytic Levi-flat subset in $\mathbb{P}^{N}$. Let $\sigma: \mathbb{C}^{N+1} \rightarrow \mathbb{P}^{N}$ be the natural projection. Suppose that $H$ is a real-analytic subvariety of $\mathbb{P}^{N}$. Define the set $\tau(H)$ to be the set of points $z \in \mathbb{C}^{N+1}$ such that $\sigma(z) \in H$ or $z=0$. A real analytic subvariety $H \subset \mathbb{P}^{N}$ is said to be algebraic if $H=\sigma(V)$ for some real algebraic complex cone $V$ in $\mathbb{C}^{N+1}$. A set $V$ is a complex cone when $p \in V$ implies $\lambda p \in V$ for all $\lambda \in \mathbb{C}$.

The following construction offers several examples of Levi-flat subsets in $\mathbb{P}^{N}$.

Proposition 3.7. $\left[2, \quad\right.$ Proposition 6.1] Let $X \subset \mathbb{P}^{N}$ be an irreducible $(n+1)$-dimensional algebraic variety, $R$ be a rational function in $X$ and $C \subset \mathbb{P}^{1}$ be a real algebraic one-dimensional subvariety. Then the set $\overline{R^{-1}(C)}$ is a real algebraic Levi-flat subset of Levi dimension $n$ whose $\mathrm{\imath -complexification}$ is $X$.

When we add the hypothesis that the Levi-flat subset is invariant by a singular holomorphic foliation in the ambient space, we can state a reciprocal result.

Proposition 3.8. ([2, Proposition 6.3]) Let $\mathscr{F}$ be a singular holomorphic foliation in $\mathbb{P}^{N}$ tangent to a real analytic Levi-flat subset $H$ of Levi dimension $n$. Suppose that $H$ is coherent and its -complexification extends to an algebraic subvariety $H^{\imath}$ in $\mathbb{P}^{N}$. If $\mathscr{F}^{\imath}$ has a rational first integral $R$, then there exists a real algebraic one-dimensional subvariety $C \subset \mathbb{P}^{1}$ such that $\overline{H_{\text {reg }}} \subset \overline{R^{-1}(C)}$.

Now, since $H$ is coherent, the intrinsic complexification $H^{\imath}$ is well-defined as a complex subvariety in a neighborhood of $H$. Our aim is to extend $H^{\imath}$ to an algebraic subvariety in $\mathbb{P}^{N}$. To get this, we use the following extension theorem.

Theorem 3.9. (Chow [7]) Let $Z \subset \mathbb{P}^{N}$ be a complex algebraic subvariety of dimension $k$ and $V$ be a connected neighborhood of $Z$ in $\mathbb{P}^{N}$. Then any complex 
analytic subvariety of dimension higher than $N-k$ in $V$ that intersects $Z$ extends algebraically to $\mathbb{P}^{N}$.

Under certain hypotheses, we can prove that the $\imath$-complexification $H^{\imath}$ can be extended to $\mathbb{P}^{N}$.

Proposition 3.10. Let $H \subset \mathbb{P}^{N}, N \geq 3$, be an irreducible coherent real analytic Levi-flat subset of Levi dimension $n$ such that $n>N / 2$. If the Levi foliation $\mathscr{L}$ has a quasi-invariant complex algebraic subvariety of complex dimension $n$, then $H^{\imath}$ extends algebraically to $\mathbb{P}^{N}$.

Proof. Denote by $L$ such quasi-invariant algebraic complex subvariety with $\operatorname{dim}_{\mathbb{C}} L=n-1$. Since $L$ algebraic with $L \subset H^{\imath}$ and $\operatorname{dim}_{\mathbb{C}} H^{\imath}=n+1>N-(n-1)$, we can apply Theorem 3.9 to prove that $H^{\imath}$ extends algebraically to $\mathbb{P}^{N}$.

To end this section, we shall prove the following proposition.

Proposition 3.11. Let $H \subset \mathbb{P}^{N}$ be an irreducible coherent real analytic Leviflat subset of Levi dimension $n$ invariant by an $n$-dimensional singular holomorphic foliation $\mathscr{F}$ in $\mathbb{P}^{N}$. Suppose that the -complexification $H^{\imath}$ extends to an algebraic variety in $\mathbb{P}^{N}$. If the Levi-foliation $\mathcal{L}$ has infinitely many quasi-invariant algebraic subvarieties of complex dimension $n-1$. Then, either the foliation $\mathscr{F}^{\iota}=\left.\mathscr{F}\right|_{H^{\imath}}$ has a rational first integral in $H^{\imath}$, or $\mathscr{F}^{\iota}$ is a pull-back of a foliation on a projective surface under a dominant rational map.

Proof. First of all, we need to desingularize the $\imath$-complexification $H^{\imath}$. According to Hironaka desingularization theorem, there exist a complex manifold $\widetilde{H^{\imath}}$ and a proper bimeromorphic morphism $\pi: \widetilde{H^{\imath}} \rightarrow H^{\imath}$ such that

1. $\pi: \widetilde{H^{\imath}} \backslash\left(\pi^{-1}\left(\operatorname{Sing}\left(H^{\imath}\right)\right) \rightarrow H^{\imath} \backslash \operatorname{Sing}\left(H^{\imath}\right)\right.$ is a biholomorphism,

2. $\pi^{-1}\left(\operatorname{Sing}\left(H^{\imath}\right)\right)$ is a simple normal crossing divisor.

Since $H^{\imath}$ is compact then ${\widetilde{H^{\imath}}}^{\imath}$ is too. We lift $\mathscr{F}^{\imath}$ to an $n$-dimensional singular holomorphic foliation $\widetilde{\mathscr{F}^{\imath}}$ on $\widetilde{H^{\imath}}$. Since $\operatorname{dim}_{\mathbb{C}} \widetilde{H^{\imath}}=n+1$, we have $\widetilde{\mathscr{F}^{\imath}}$ has codimension one on $\widetilde{H^{\imath}}$ and the tangency condition between $\mathscr{F}^{\imath}$ and $H$ implies that $\mathscr{F}^{\imath}$ has infinitely many quasi-invariant closed subvarieties (these are algebraic and of codimension one in $\widetilde{H^{\imath}}$ ). Thus the same holds for $\widetilde{\mathscr{F}}$. By Theorem 2.3, either $\widetilde{\mathscr{F}}^{\imath}$ has a rational first integral or there exist a dominant rational map $\tilde{\rho}: \widetilde{H^{\imath}} \rightarrow-\rightarrow$, where $Y$ is a projective complex surface, $\mathcal{G}$ is a foliation by curves on $Y$ and $\widetilde{\mathscr{F}}^{\imath}=\tilde{\rho}^{*}(\mathcal{G})$. If $\widetilde{\mathscr{F}}^{\imath}$ admits a rational first integral in $\widetilde{H^{\imath}}$, then all leaves of $\widetilde{\mathscr{F}} \imath$ are compact and so their $\pi$-images are compact leaves of $\mathscr{F}^{\imath}$ in $H^{\imath}$. Applying Gómez-Mont's theorem [12], we have that there exists a one-dimensional projective manifold $S$ and a rational map $f: H^{\imath} \rightarrow S$ whose fibers contain the leaves of $\mathscr{F}^{\imath}$. A rational first integral is obtained by composing $f$ with any non-constant rational map $r: S-\rightarrow \mathbb{P}^{1}$. If $\widetilde{\mathscr{F}}^{\imath}$ is 
a pull-back of a foliation $\mathcal{G}$ on a projective complex surface $Y$ under a dominant rational map $\tilde{\rho}: \widetilde{H}^{\imath} \rightarrow Y$ then $\mathscr{F}^{\imath}$ is the pull-back of $\mathcal{G}$ under $\rho:=\tilde{\rho} \circ \pi^{-1}: H^{\imath}-\rightarrow Y$, since $\pi$ is a birational map.

\section{Proof of Theorem 1.1}

With all the above results, we can prove Theorem 1.1.

Theorem 4.1. Let $H \subset \mathbb{P}^{N}, N \geq 3$, be an irreducible real analytic Levi-flat subset of Levi dimension $n$ invariant by an $n$-dimensional singular holomorphic foliation $\mathscr{F}$ on $\mathbb{P}^{N}$. Suppose that $H$ is coherent and $n>N / 2$. If the Levi foliation has infinitely many quasi-invariant subvarieties of complex dimension $n$, then there exists a unique projective subvariety $X$ of complex dimension $n+1$ containing $H$ such that either there exists a rational map $R: X \rightarrow \rightarrow \mathbb{P}^{1}$, and real algebraic curve $C \subset \mathbb{P}^{1}$ such that $\overline{H_{\text {reg }}} \subset \overline{R^{-1}(C)}$ or there exists a dominant rational map $\rho: X-\rightarrow Y$ on a projective surface $Y$ and a semianalytic Levi-flat subset $T \subset Y$ such that $\overline{H_{\text {reg }}} \subset \overline{\rho^{-1}(T)}$.

Proof. By Proposition 3.6, there exist an open neighborhood $V \subset \mathbb{P}^{N}$ of $H$ and a unique irreducible complex subvariety $H^{\imath}$ of $V$ of complex dimension $n+1$ containing $H$. The Proposition 3.5 implies that $H^{\imath}$ is invariant by $\mathscr{F}$ and moreover it extends algebraically to $\mathbb{P}^{N}$ by Proposition 3.10 . We denote $\mathscr{F}^{\imath}:=\left.\mathscr{F}\right|_{H^{\imath}}$ the restrict foliation to $H^{\imath}$. Observe now that $\mathscr{F}^{\imath}$ is a foliation of codimension one on $H^{\imath}$ which admit infinitely many quasi-invariant subvarieties of complex dimension $n-1$. Therefore, either $\mathscr{F}^{\iota}$ has a rational first integral in $H^{\imath}$, or $\mathscr{F}^{\iota}$ is a pull-back of a foliation on a projective surface under a dominant rational map by Proposition 3.11.

If $\mathscr{F}^{2}$ has a first integral $R$ then there exists a real algebraic curve $C \subset \mathbb{P}^{1}$ such that $\overline{H_{\text {reg }}} \subset \overline{R^{-1}(C)}$ by Proposition 3.8. Now if we assume that $\mathscr{F}^{\imath}$ is a pull-back of a foliation $\mathcal{G}$ on a projective complex surface $Y$ under a dominant rational map $\rho: H^{\imath}-\rightarrow Y$. Then we can take $X=H^{\imath}$. Let us prove that there exists a semianalytic Levi-flat subset $T \subset Y$. Indeed, let $z \in H_{\text {reg }} \backslash \operatorname{Ind}(\rho)$ (here $\operatorname{Ind}(\rho)$ denotes the indeterminacy set of $\rho$ ). Then there exists a neighborhood $U \subset H^{\imath} \backslash \operatorname{Ind}(\rho)$ of $z$ and a non-singular real analytic curve $\gamma:(-\varepsilon, \varepsilon) \rightarrow U$ such that $\gamma(0)=z,\{\gamma\} \subset H_{\text {reg }}$, and such that $\gamma$ is transverse to the Levi foliation $\mathscr{L}$ on $H_{\text {reg }}$. Let $L_{\gamma(t)}$ be the leaf of $\mathscr{L}$ through $\gamma(t)$. Since $L_{\gamma(t)}$ is also a leaf of $\mathscr{F}^{\imath}$ and $\mathscr{F}^{\imath}=\rho^{*}(\mathcal{G})$, then $\rho\left(L_{\gamma(t)}\right)$ is a leaf of $\mathcal{G}$. Let us denote $A_{t}=\overline{\rho\left(L_{\gamma(t)}\right)} \subset Y$ and define

$$
T_{z}:=\bigcup_{t \in(-\varepsilon, \varepsilon)} A_{t} \subset V_{z},
$$

where $V_{z}$ is a neighborhood of $T_{z}$ on $Y$. Note that $T_{z}$ is a union of complex subvarieties parametrized by $t$ such that each $A_{t}$ contains leaves of $\mathcal{G}$, thus $T_{z}$ is a semian- 
alytic Levi-flat subset on $V_{z}$. These local constructions are sufficiently canonical to be patched together when $z$ varies on $H_{\text {reg }}$ : if $T_{z_{1}} \subset V_{z_{1}}$ and $T_{z_{2}} \subset V_{z_{2}}$ are as above, with $V_{z_{1}} \cap V_{z_{2}} \neq \varnothing$, then $T_{z_{1}} \cap V_{z_{1}} \cap V_{z_{2}}$ and $T_{z_{2}} \cap V_{z_{1}} \cap V_{z_{2}}$ have some common leaves of $\mathcal{G}$ because $\mathcal{G}$ is a global foliation defined on $Y$, so $T_{z_{1}}$ and $T_{z_{2}}$ can be glued by identifying these leaves. In this way, we get a semianalytic Levi-flat subset $T$ in $Y$.

Finally, we assert that $\overline{H_{\text {reg }}} \subset \overline{\rho^{-1}(T)}$. In fact, let $w \in \overline{H_{\text {reg }}}$, then there exists a sequence $z_{k} \rightarrow w, z_{k} \in H_{\text {reg }}$, so $\rho\left(z_{k}\right) \in T$ which imply that $z_{k} \in \rho^{-1}(T)$ and $w \in \overline{\rho^{-1}(T)}$. This finishes the proof.

\section{Proof of Corollary 1.2}

Corollary 5.1. Let $H \subset \mathbb{P}^{N}, N \geq 3$, be an irreducible coherent real analytic Levi-flat hypersurface invariant by a codimension one holomorphic foliation $\mathscr{F}$ on $\mathbb{P}^{N}$. If the Levi foliation has infinitely many quasi-invariant complex hypersurfaces,

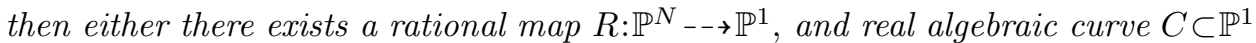
such that $\overline{H_{\text {reg }}} \subset \overline{R^{-1}(C)}$ or there exists a dominant rational map $\rho: \mathbb{P}^{N} \rightarrow Y$ on a projective complex surface $Y$ and a semianalytic Levi-flat subset $T \subset Y$ such that $\overline{H_{\text {reg }}} \subset \overline{\rho^{-1}(T)}$.

Proof. If $H$ is an irreducible real analytic Levi-flat hypersurface in $\mathbb{P}^{N}, N \geq 3$, then the Levi dimension of $H$ is $N-1$. Moreover

$$
N-1>N / 2 \Longleftrightarrow N>2 \text {. }
$$

Thus, we can apply Theorem 1.1 to $H$, so there exist a unique projective subvariety $X$ of complex dimension $N$ containing $H$ such that either there exists a rational map $R: X-\rightarrow \mathbb{C}$, and real algebraic curve $C \subset \mathbb{C}$ such that $\overline{H_{r e g}} \subset \overline{R^{-1}(C)}$ or there exists a dominant rational map $\rho: X-\rightarrow Y$ on a projective complex surface $Y$ and a semianalytic Levi-flat subset $T \subset Y$ such that $\overline{H_{\text {reg }}} \subset \overline{\rho^{-1}(T)}$. Since $X \subset \mathbb{P}^{N}$ has complex dimension $N$, we must have $X=\mathbb{P}^{N}$ and hence we conclude the proof.

\section{Examples}

Example 6.1. We give an example of a real analytic Levi-flat hypersurface in $\mathbb{P}^{3}$ where Theorem 1.1 applies. Let

$$
H=\left\{\left[z_{0}: z_{1}: z_{2}: z_{3}\right] \in \mathbb{P}^{3}: z_{0} z_{1} \bar{z}_{2} \bar{z}_{3}-z_{2} z_{3} \bar{z}_{0} \bar{z}_{1}=0\right\},
$$

then $H$ is Levi-flat because it is foliated by the complex hypersurfaces

$$
z_{0} z_{1}=c z_{2} z_{3}, \quad \text { where } \quad c \in \mathbb{R} \text {. }
$$


Let $\mathscr{F}$ be the codimension one holomorphic foliation on $\mathbb{P}^{3}$ of degree two defined by

$$
\omega=z_{1} z_{2} z_{3} d z_{0}+z_{0} z_{2} z_{3} d z_{1}-z_{0} z_{1} z_{3} d z_{2}-z_{0} z_{1} z_{2} d z_{3},
$$

then $\mathscr{F}$ has a rational first integral $R: \mathbb{P}^{3}-\rightarrow \mathbb{P}^{1}$ given by

$$
R\left[z_{0}: z_{1}: z_{2}: z_{3}\right]=\left[z_{0} z_{1}: z_{2} z_{3}\right] .
$$

Since the leaves of $\left.\mathscr{F}\right|_{H}$ coincide with the leaves of the Levi foliation (3), $H$ must be invariant by $\mathscr{F}$. On the other hand, note that $H=\overline{R^{-1}(C)}$, where

$$
C=\left\{[t: u] \in \mathbb{P}^{1}: t \bar{u}-u \bar{t}=0\right\} .
$$

Example 6.2. In the following example, we construct a real analytic Levi-flat hypersurface $H$ in $\mathbb{P}^{3}$ that is not a pull-back of a Levi-flat hypersurface of $\mathbb{P}^{2}$ under a rational map, furthermore, $H$ also is not a pull-back of a real algebraic curve under a meromorphic function.

Consider $z=\left(z_{0}, z_{1}, z_{2}, z_{3}\right), \bar{z}=\left(\bar{z}_{0}, \bar{z}_{1}, \bar{z}_{2}, \bar{z}_{3}\right)$ and

$$
F(z, \bar{z})=\operatorname{det}\left(\begin{array}{cccccc}
z_{0} & z_{1} & z_{2} & z_{3} & 0 & 0 \\
0 & z_{0} & z_{1} & z_{2} & z_{3} & 0 \\
0 & 0 & z_{0} & z_{1} & z_{2} & z_{3} \\
\bar{z}_{0} & \bar{z}_{1} & \bar{z}_{2} & \bar{z}_{3} & 0 & 0 \\
0 & \bar{z}_{0} & \bar{z}_{1} & \bar{z}_{2} & \bar{z}_{3} & 0 \\
0 & 0 & \bar{z}_{0} & \bar{z}_{1} & \bar{z}_{2} & \bar{z}_{3}
\end{array}\right)
$$

Define $H=\left\{\left[z_{0}: z_{1}: z_{2}: z_{3}\right] \in \mathbb{P}^{3}: F(z, \bar{z})=0\right\}, H$ is a real analytic hypersurface well defined since $F$ is a bihomogeneous polynomial of bi-degree $(3,3)$. Moreover, $H$ is Levi-flat, because it is foliated by the complex hyperplanes

$$
z_{0}+c z_{1}+c^{2} z_{2}+c^{3} z_{3}=0, \quad \text { where } \quad c \in \mathbb{R} .
$$

Let $\mathscr{W}$ be the codimension one holomorphic 3 -web on $\mathbb{P}^{3}$ given by the implicit differential equation $\Omega=0$,

$$
\Omega=\operatorname{det}\left(\begin{array}{cccccc}
z_{0} & z_{1} & z_{2} & z_{3} & 0 & 0 \\
0 & z_{0} & z_{1} & z_{2} & z_{3} & 0 \\
0 & 0 & z_{0} & z_{1} & z_{2} & z_{3} \\
d z_{0} & d z_{1} & d z_{2} & d z_{3} & 0 & 0 \\
0 & d z_{0} & d z_{1} & d z_{2} & d z_{3} & 0 \\
0 & 0 & d z_{0} & d z_{1} & d z_{2} & d z_{3}
\end{array}\right)
$$

Since the leaves of $\left.\mathscr{W}\right|_{H}$ and $\mathscr{L}$ are the same, we get $H$ is invariant by $\mathscr{W}$.

Now, we prove that $H$ is not a pull-back of a Levi-flat hypersurface of $\mathbb{P}^{2}$. To prove this fact, we use the following result of [13, Proposition 4.4]: 
Proposition 6.3. Let $\omega_{1}, \omega_{2}$ and $\omega_{3}$ be independent germs of integrable 1 -forms at $\left(\mathbb{C}^{3}, 0\right)$ with singular sets of codimension at least two. Suppose that there exists a non-zero holomorphic 2-form $\eta$, locally decomposable outside its singular set, that is tangent to each $\omega_{i}$, for $i=1,2,3$. Then $\omega_{1}, \omega_{2}$ and $\omega_{3}$ define foliations that are in a pencil. Furthermore, $\eta$ is integrable, defining the axis foliation of this pencil.

Suppose by contradiction that $H$ is a pull-back of a Levi-flat hypersurface under a dominant rational map $\rho: \mathbb{P}^{3} \rightarrow \rightarrow \mathbb{P}^{2}$. Then pick a point $p \in U_{0}$, where $U_{0}$ is an open subset in $\mathbb{P}^{3}$ such that $\left.\rho\right|_{U_{0}}: U_{0} \subset \mathbb{C}^{3} \rightarrow \mathbb{C}^{2}$ is a holomorphic submersion. We may have needed to perhaps move to yet another point $p^{\prime} \in U_{0}$ such that $U_{0}$ does not intersect the discriminant set of the web $\mathscr{W}$. We set $p=p^{\prime}$ and works in a neighborhood of $U_{0}$. Therefore, the germ of $\mathscr{W}$ at $p$ is a decomposable 3 -web, defined by the superposition of three independent foliations $\mathscr{F}_{1}, \mathscr{F}_{2}$, and $\mathscr{F}_{3}$. We can assume that these foliations are defined by independent germs of integrable 1-forms $\omega_{1}, \omega_{2}$, and $\omega_{3}$ respectively. Since $H$ is given by a pull-back, all the leaves of $\mathscr{L}$ and, hence the leaves of $\mathscr{W}$ in $H \cap U_{0}$ are tangent to the fibers of $\left.\rho\right|_{U_{0}}$, these fibers define a non-zero holomorphic 2 -form $\eta_{\rho}$ that is tangent to each $\omega_{i}$, for $i=1,2,3$. Then, according to Proposition $6.3, \omega_{1}, \omega_{2}$, and $\omega_{3}$ define foliations that are in a pencil, an absurd. Hence, the assertion is proved.

Now we assert that $H$ is not a pull-back of a real algebraic curve under a meromorphic function. In fact, $H$ is a Levi-flat hypersurface in $\mathbb{P}^{3}$ such that there does not exist a point contained in infinitely many leaves of $\mathscr{L}$, because, the leaves of $\mathscr{L}$ are given by the equation (4) and through at a point only pass three leaves. If $H$ is defined by a pull-back of a meromorphic function, there has to exist a point $p$ of indeterminacy since the dimension is at least 2 . Then through at $p$ pass infinitely many leaves of $\mathscr{L}$. Since $H$ does not satisfy this property, we finish the proof of the assertion.

Acknowledgments. The authors wish to express gratitude to Maria Aparecida Soares Ruas (ICMC - USP, São Carlos) and Judith Brinkschulte (Universität Leipzig) for many valuable conversations and suggestions.

\section{References}

1. Beltrán, A., Fernández-PÉrez, A. and Neciosup, H., Existence of dicritical singularities of Levi-flat hypersurfaces and holomorphic foliations, Geom. Dedic. (2017). doi:10 . 1007/s10711-017-0303-4

2. Bretas, J., Fernández-PÉrez, A. and Mol, R., Holomorphic foliations tangent to Levi-flat subsets, J. Geom. Anal. 29 (2019), 1407. doi:10.1007/s12220-018-0043-1 
3. Brunella, M., Singular Levi-flat hypersurfaces and codimension one foliations, Ann. Sc. Norm. Super. Pisa, Cl. Sci. (5) VI (2007), 661-672.

4. Cartan, E., Sur la géométrie pseudo-conforme des hypersurfaces de l'espace de deux variables complexes, Ann. Mat. Pura Appl. 11 (1933), 17-90.

5. Cerveau, D. and Sad, P., Fonctions et feuilletages Levi-flat. ètude locale, Ann. Sc. Norm. Super. Pisa, Cl. Sci. (5) 3 (2004), 427-445.

6. Cervenu, D. and Lins Neto, A., Local Levi-flat hypersurfaces invariants by a codimension one holomorphic foliation, Am. J. Math. 133 (2011), 677-716. doi:10.1353/ajm. 2011.0018

7. Chow, W. L., On meromorphic maps of algebraic varieties, Ann. Math. 2 (1969), 391-403.

8. Fernández-PÉREz, A., On Levi-flat hypersurfaces with generic real singular set, $J$. Geom. Anal. 23 (2013), 2020. doi:10.1007/s12220-012-9317-1

9. Fernández-PÉREz, A., Levi-flat hypersurfaces tangent to projective foliations, J. Geom. Anal. 24 (2014), 1959. doi:10.1007/s12220-013-9404-y

10. Fernández-PÉrez, A., Mol, R. and Rosas, R., On singular real analytic Levi-flat foliations, Asian J. Math. (2020). doi:10 .4310/A JM . 2020 .v24 .n6 .a4

11. Guaraldo, F., Macrì, P. and Tancredi, A., Topics on real analytic spaces, Springer, Berlin, 2013.

12. Gómez-Mont, X., Integrals for holomorphic foliations with singularities having all leaves compact, Ann. Inst. Fourier 39 (1989), 451-458.

13. JuncA, D. and MoL, R., Holomorphic vector fields tangent to foliations in dimension three, An. Acad. Brasil. Ciênc. 93 (2021). doi:10 . 1590/0001-3765202020181390

14. LeBL, J., Algebraic Levi-flat hypervarieties in complex projective space, J. Geom. Anal. 22 (2012), 410. doi:10.1007/s12220-010-9201-9

15. LeBL, J., Singular set of a Levi-flat hypersurface is Levi-flat, Math. Ann. 355 (2013), 1177. doi:10.1007/s00208-012-0821-1

16. LeBL, J., Singular Levi-flat hypersurfaces in complex projective space induced by curves in the Grassmannian, Int. J. Math. 26, 1550036 (2015), 17 pp.

17. Narasimhan, R., Introduction to the theory of analytic spaces, Lectures Notes in Mathematics, Springer, Berlin Heidelberg, 1966, doi:10 .1007/BFb0077071

18. Ni, L. and Wolfson, J., The Lefschetz theorem for CR submanifolds and the nonexistence of real analytic Levi flat submanifolds, Commun. Anal. Geom. 11 (2003), $553-564$.

19. Pereira, J. V. and Spicer, C., Hypersurfaces quasi-invariant by codimension one foliations, Math. Ann. (2019). doi:10.1007/s00208-019-01833-4

20. Pinchuk, S., Shafikov, R. and Sukhov, A., On dicritical singularities of Levi-flat sets, Ark. Mat. 56 (2018), 395-408. doi:10.4310/ARKIV . 2018 .v56.n2 . a12

21. Shafikov, R. and Sukhov, A., Germs of singular Levi-flat hypersurfaces and holomorphic foliations, Comment. Math. Helv. 90 (2015), 479-502.

22. Sukhov, A., Levi-flat world: a survey of local theory, Ufimsk. Mat. Zh. 9 (2017), $172-185$. 
Andrés Beltrán

Dpto. Ciencias - Sección Matemáticas Pontifícia Universidad Católica del Perú

Av. Universitaria 1801, San Miguel

Lima PE-32

Peru

abeltra@pucp.pe

Arturo Fernández-Pérez

Departamento de Matemática - ICEX

Universidade Federal de Minas Gerais, UFMG

Av. Antônio Carlos 6627

Belo Horizonte-MG BR-31270-901

Brasil

fernandez@ufmg.br

Received June 16, 2020

\section{Hernán Neciosup}

Dpto. Ciencias - Sección Matemáticas Pontifícia Universidad Católica del Perú Av. Universitaria 1801, San Miguel Lima PE-32

Peru

hneciosup@pucp.pe 